\title{
Maximum Power, Ecological Function and Efficiency of an Irreversible Carnot Cycle. A Cost and Effectiveness Optimization
}

\author{
G. Aragón-González*, A. Canales-Palma, A. León-Galicia, and J. R. Morales-Gómez \\ PDPA. UAM- Azcapotzalco. Av. San Pablo \# 180. Col. Reynosa. \\ Azcapotzalco, 02800, México \\ (Received on 31 March, 2008)
}

\begin{abstract}
In this work we include, for the Carnot cycle, irreversibilities of linear finite rate of heat transfers between the heat engine and its reservoirs, heat leak between the reservoirs and internal dissipations of the working fluid. A first optimization of the power output, the efficiency and ecological function of an irreversible Carnot cycle, with respect to: internal temperature ratio, time ratio for the heat exchange and the allocation ratio of the heat exchangers; is performed. For the second and third optimizations, the optimum values for the time ratio and internal temperature ratio are substituted into the equation of power and, then, the optimizations with respect to the cost and effectiveness ratio of the heat exchangers are performed. Finally, a criterion of partial optimization for the class of irreversible Carnot engines is herein presented.
\end{abstract}

Keywords: Internal and external irreversibilities; Heat engines; Finite time and size thermodynamics; Cost and effectiveness optimization

\section{INTRODUCTION}

The thermal efficiency of a reversible Carnot cycle is an upper limit of efficiency for heat engines. In according to classical thermodynamics, the Carnot efficiency is:

$$
\eta_{C}=1-\frac{T_{L}}{T_{H}}
$$

where $T_{L}$ and $T_{H}$ are the temperatures of the hot and cold reservoirs between which the heat engine operates. The thermal efficiency $\eta_{C}$ can only be achieved through the infinitely slow process required by thermodynamic equilibrium. Therefore, it is not possible to obtain a certain amount of power output by using heat exchangers with finite heat transfer areas. Thus, the thermal efficiency given in equation (1.1) does not have great significance and is a poor guide for the performances of real heat engines.

A more realistic upper bound could be placed on the efficiency of a heat engine operating at its maximum power point; the so-called CA efficiency (Curzon-Alhborn [1]):

$$
\eta_{C A}=1-\sqrt{\frac{T_{L}}{T_{H}}}
$$

where the only source of irreversibility in the engine is a linear finite rate heat transfer between the working fluid and its two heat reservoirs.

Real heat engines are complex devices. Besides the irreversibility of finite-rate heat transfer in finite time taken into account in the Curzon-Ahlborn engine (CA-engine), there are also other sources of irreversibility, such as heat leaks, dissipative processes inside the working fluid and so on. Thus, it is necessary to investigate more comprehensively the influence of finite-rate heat transfer together with other major irreversibilities on the performance of heat engines. For this aim,

*e-mail: gag@correo.azc.uam.mx. we must consider general irreversible Carnot engines including three major irreversibilities, which often exist in heat engines, and use it to optimize the performance of an irreversible Carnot engine for several objective functions.

In the past decade some new models of irreversible Carnot engines which include other irreversibilities, besides thermal resistance, have been established: heat leak and internal dissipations of the working fluid (see [2-10] and included references there). Nevertheless, there are other parameters involved in the performance and optimization of an irreversible Carnot cycle; for instance, the allocation ratio of the heat exchangers, cost and effectiveness ratio of the heat exchangers and so on (see [8], [9] and [14]).

In the optimization of Carnot cycles, including those irreversibilities, have appeared four objective functions: power, efficiency, ecological and entropy generation. The maximum power and efficiency have been obtained in [4], [5] and [10]. The maximum ecological function was obtained in [12] for the CA-engine and in form more general in [7]. Bejan [13] has considered the minimization of the entropy generation. In general, these optimizations were performed with respect to only one characteristic parameter: internal temperature ratio. In the first analysis of the CA-engine the time ratio of heat transfer from hot to cold side was considered, but in further works this ratio was not taken into account (see [2] and [6] for more details). In [17] this ratio was taken into account as a characteristic parameter of the engine and we found that the time allocation of heat transfer between the hot to cold sides is the same for power maximum and efficiency maximum. On the other hand, [13] has performed the optimization, also, with respect to other parameter: the allocation ratio of the heat exchangers; and [14] and [9] have considered as parameters the cost and effectiveness ratio of the heat exchangers for the CAengine.

This paper is organized as follows. In the section 2 the relations for the dimensionless power, efficiency, entropy generation and ecological function of a class of irreversible Carnot engines are presented. In the section 3, the optimal analytical expressions for the efficiencies corresponding to power 
and ecological function; and maximum efficiency are shown. In section 4, the optimum values for the time ratio and internal temperature ratio are substituted in the expression for dimensionless power. Then a second and third optimizations of dimensionless power, are performed with respect to the cost and effectiveness ratio of the heat exchangers. In the section of Conclusions, a criterion of partial optimization for power, ecological function, efficiency and entropy generation is presented.

\section{IRREVERSIBLE CARNOT ENGINE.}

In considering the class of irreversible Carnot engines (see [2]) shown in Fig. 1, which satisfy the following five conditions:

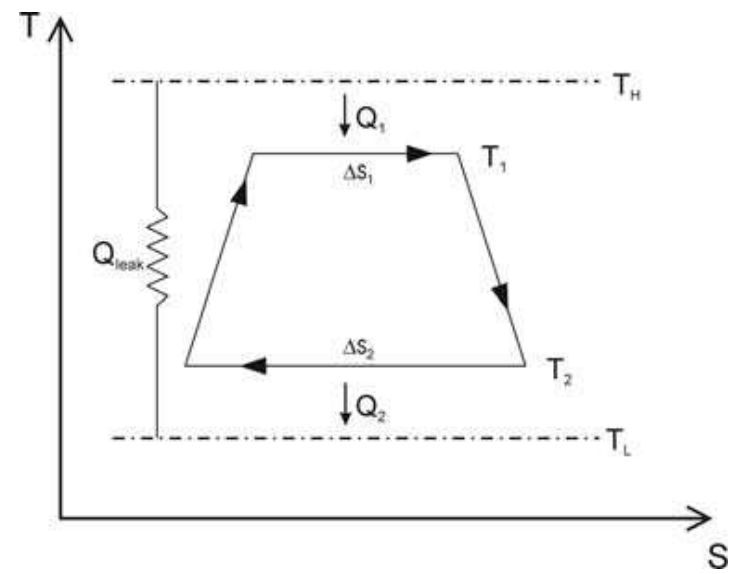

FIG. 1: A Carnot cycle with heat leak, finite-rate heat transfer and internal dissipations of the working fluid.

(i) The cycle of the engine consists of two isothermal and two adiabatic processes. The temperatures of the working fluid in the hot and cold isothermal processes are, respectively, $T_{1}$ and $T_{2}$, and the times of the two isothermal processes are, respectively, $t_{H}$ and $t_{L}$. The temperatures of the hot and cold heat reservoirs are, respectively, $T_{H}$ and $T_{L}$.

(i) There is thermal resistance between the working fluid and the heat reservoirs.

(ii) There is a heat lost $Q_{\text {leak }}$ from the hot reservoir to the cold reservoir [13]. In real engines heat leaks are unavoidable, there are many features of an actual power plant which fall under that kind of irreversibility, such as the heat lost through the walls of a boiler, a combustion chamber, or a heat exchanger, and heat flow through the cylinder walls of an internal combustion engine, and so on.

(iii) All heat transfer is assumed to be linear in temperature differences, that is, Newtonian.

(iv) Besides thermal resistance and heat loss, there are other irreversibilities in the cycle, the internal irreversibilities. For many devices such as gas turbines, automotive engines, and thermoelectric generator, there are other loss mechanisms, like friction or generators losses, etc. that play an important role, but are hard to model in detail. Some authors use the compressor (pump) and turbine isentropic efficiencies to model the internal loss in the gas turbines or steam plants. Others, in Carnot cycles, use simply one parameter to describe the internal losses. Such a parameter is associated with the entropy produced inside the engine during a cycle. Specifically, this parameter makes the Claussius inequality becomes an equality (for details see [2]):

$$
\frac{Q_{2}}{T_{2}}-I \frac{Q_{1}}{T_{1}}=0
$$

where $I=\frac{\Delta S_{2}}{\Delta S_{1}} \geq 1([4])$.

Thus, the irreversible Carnot engine operates with fixed time $t$ allowed for each cycle. The heat leakage $Q_{\text {leak }}$ is ([13]):

$$
Q_{\text {leak }}=K\left(T_{H}-T_{L}\right) t
$$

The heats $Q_{H}, Q_{L}$ transferred from the hot-cold reservoirs are given by:

$$
\begin{gathered}
Q_{H}=Q_{1}+Q_{\text {leak }}=\alpha\left(T_{H}-T_{1}\right) t_{H}+K\left(T_{H}-T_{L}\right) t \\
Q_{L}=Q_{2}+Q_{\text {leak }}=\beta\left(T_{2}-T_{L}\right) t_{L}+K\left(T_{H}-T_{L}\right) t
\end{gathered}
$$

where $\alpha, \beta$ and $K$ are the thermal conductances and $t_{H}, t_{L}$ are the time for the heat transfer in the isothermal branches, respectively. The connecting adiabatic branches are often assumed to proceed in negligible time ([3]), such that the cycle contact total time $t$ is [11]:

$$
t=t_{H}+t_{L}
$$

By first law and combining equations (2.2) and (2.3) we obtain:

$$
\begin{gathered}
W=Q_{1}(1-I x)=\frac{T_{H}(1-I x)\left(1-\frac{\mu}{x}\right)}{\frac{1}{\alpha t_{H}}+\frac{I}{\beta t_{L}}} \\
Q_{H}=Q_{1}+Q_{\text {leak }}=\frac{W}{1-I x}+K\left(T_{H}-T_{L}\right) t
\end{gathered}
$$

where $\mu=\frac{T_{L}}{T_{H}}$. And $x=\frac{T_{2}}{T_{1}}$ is a characteristic parameter of the engine.

Now, the equation (2.4) gives us the total time of the cycle, so it can be parametrized as:

$$
t_{H}=y t ; t_{L}=(1-y) t
$$

where $y=\frac{t_{H}}{t}=\frac{t_{H}}{t_{H}+t_{L}}$ is other characteristic parameter of the engine.

Another parameter is the allocation of the exchangers heat [13]. The thermal conductances can be written as:

$$
\alpha=U A_{H} ; \beta=U A_{L}
$$

where $U$ is overall heat transfer coefficient and $A_{H}$ and $A_{L}$ are the available areas for heat transfer. Then, an approach might 
be to suppose that $U$ is fixed, the same for the hot side and the cold side heat exchangers, and that the area $A$ can be allocated between both. The optimization problem is then selected, besides of the optimum temperature ratio and the time ratio, as the best allocation ratio. To take $U A$ as a fixed value can be justified in terms of the area purchased, and the fixed running costs and capital costs that altogether determine the overall heat transfer coefficient (see ([14])). Thus, for the optimization we can take:

$$
\frac{\alpha}{U}+\frac{\beta}{U}=A
$$

and parametrize it as:

$$
\begin{gathered}
\alpha=z U A ; \beta=(1-z) U A \\
\frac{\alpha}{\beta}=\frac{z}{(1-z)}
\end{gathered}
$$

Therefore, the dimensionless power output, $p=\frac{W}{A U t T_{H}}$, and the dimensionless heat transfer rate $q_{H}=\frac{Q_{H}}{A U t T_{H}}$ are (by equations (2.5) and (2.6)):

$$
\begin{array}{r}
p=\frac{z(1-z) y(1-y)(1-I x)\left(1-\frac{\mu}{x}\right)}{(1-z)(1-y)+z y I} \\
q_{H}=\frac{z(1-z) y(1-y)\left(1-\frac{\mu}{x}\right)}{(1-z)(1-y)+z y I}+L(1-\mu)
\end{array}
$$

where $L=\frac{K}{A U}$. And $z=\frac{\alpha}{U A}$ is the third characteristic parameter of the engine. The thermal efficiency is given by:

$\eta=\frac{z(1-z) y(1-y)(1-I x)\left(1-\frac{\mu}{x}\right)}{z(1-z) y(1-y)\left(1-\frac{\mu}{x}\right)+L(1-\mu)((1-z)(1-y)+z y I)}$

The entropy-generation rate, $s_{g e n}=\frac{S_{g e n}}{A U t T_{H}}$, multiplied by the temperature of the cold side, give us a dimensionless function $\sigma$, which is (equations $(2.9,2.10)$ ):

$$
\sigma=T_{L} s_{g e n}=T_{L}\left(\frac{q_{H}-p}{T_{L}}-\frac{q_{H}}{T_{H}}\right)=q_{H}(1-\mu)-p
$$

so,

$$
\sigma=\frac{z(1-z) y(1-y)\left(1-\frac{\mu}{x}\right)(I x-\mu)}{(1-z)(1-y)+z y I}+L(1-\mu)^{2}
$$

Finally, the ecological function [12], when $T_{L}$ is the environmental temperature, is:

$$
\varepsilon=p-\sigma=p \frac{2 I x-1-\mu}{I x-1}+L(1-\mu)
$$

then,

$$
\varepsilon=\frac{z(1-z) y(1-y)\left(1-\frac{\mu}{x}\right)(1-2 I x)}{(1-z)(1-y)+z y I}+L(1-\mu)
$$

when $I=1$ and $L=0$ the expressions for the CA-engine are obtained.

\section{MAXIMUM POWER, ECOLOGICAL FUNCTION AND EFFICIENCY.}

In using the equation (2.9) and the extremes conditions:

$$
\begin{aligned}
& \left.\frac{\partial p}{\partial x}\right|_{\left(x_{m p}, y_{m p}, z_{m p}\right)}=0 \\
& \left.\frac{\partial p}{\partial y}\right|_{\left(x_{m p}, y_{m p}, z_{m p}\right)}=0 ;
\end{aligned}
$$

$$
\left.\frac{\partial p}{\partial z}\right|_{\left(x_{m p}, y_{m p}, z_{m p}\right)}=0
$$

when the power reaches its maximum, $x_{m p}, y_{m p}$ and $z_{m p}$ are given by:

$$
x_{m p}=\sqrt{\frac{\mu}{I}}
$$

$$
y_{m p}=z_{m p}=\frac{1}{\sqrt[3]{I}+1}
$$

Clearly $p$ reaches its maximum in $\left(x_{m p}, y_{m p}, z_{m p}\right)$. Indeed, all the optimal points are (necessary condition):

$$
\begin{gathered}
\{z=0, y=y, x=\mu\},\left\{x=\frac{1}{I}, y=1, z=z\right\},\{y=1, z=z, x=\mu\} \\
\{y=0, z=z, x=\mu\},\left\{x=\frac{1}{I}, z=0, y=y\right\},\{y=0, z=0, x=x\} \\
\{z=1, y=1, x=x\},\{z=1, y=y, x=\mu\},\left\{x=\frac{1}{I}, z=1, y=y\right\} \\
\left\{x=\frac{1}{I}, y=0, z=z\right\},\left\{x= \pm \sqrt{\frac{\mu}{I}}, y=\frac{1}{\sqrt[3]{I}+1}, z=\frac{1}{\sqrt[3]{I}+1}\right\}
\end{gathered}
$$


By eliminating the solutions without physical meaning, we see that there is only one global extreme point given by the equations $(3.1,3.2)$. Moreover, at this extreme point maximum power is developed. Indeed, a sufficient condition for maximum power is that the eigenvalues of the Hessian $\left(\left[\left.\frac{\partial^{2} p}{\partial w \partial u}\right|_{\left(x_{m p}, y_{m p}, z_{m p}\right)}\right]_{w, u=x, y, z}\right)$ must be negative ([15]). It is clearly fulfilled since:

$$
\left[\begin{array}{ccc}
-\frac{2 I^{\frac{3}{2}}}{\sqrt{\mu}(1+\sqrt[3]{I})^{3}} & 0 & 0 \\
0 & -\frac{2(1-\sqrt{I \mu})^{2}}{\sqrt[3]{I}(1+\sqrt[3]{I})} & 0 \\
0 & 0 & -\frac{2(1-\sqrt{I \mu})^{2}}{\sqrt[3]{I}(1+\sqrt[3]{I})}
\end{array}\right]
$$

The efficiency that maximizes the power $\eta_{m p}$ is given by (see equation (2.11),

$$
\eta_{m p}=\frac{(1-\sqrt{I \mu})}{1+\frac{L(1-\mu)(\sqrt[3]{I}+1)^{3}}{(1-\sqrt{I \mu})}}
$$

The generation of entropy is minimum when $y$ and $z$ are given by equation (3.2) and $x=\frac{\mu}{\sqrt{I}}$. Nevertheless, for these values it is seen that the corresponding power does not have physical meaning. For $x=\frac{\mu}{I}(y=0$ or 1 and $z=0$ or 1$)$, makes the first term of the equation (2.12) zero. The corresponding values of $y, z$ are also without physical meaning. For $x=\mu(y=0$ or 1 and $z=0$ or 1$)$ do not have physical meaning either. Therefore, for this kind of Carnot engine, the entropy generation does not have a global minimum within the valid interval. In [16] an engine that corresponds with the kind of irreversible Carnot cycles herein presented is analyzed but the calculations leading to the minimization of entropy generation are at fault, since they do not have physical meaning. It results that the obtained power is negative! Thus, it is only possible to minimize the entropy generation partially for the variables $y, z$ and those values are given by:

$$
y_{m \sigma}=z_{m \sigma}=\frac{1}{\sqrt[3]{I}+1}
$$

In doing a analogous analysis for the ecological function, we have by the equation (2.13) that the unique extrema point of ecological function solutions with physical meaning is:

$$
x_{m e c}=\sqrt{\frac{\mu(1+\mu)}{2 I}},
$$

$$
y_{m e c}=z_{m e c}=\frac{1}{\sqrt[3]{I}+1}
$$

and newly can see that its Hessian has all its negative eingenvalues.

The efficiency that maximizes the ecological function $\eta_{\text {mec }}$ is given by (equation (2.11)):

$$
\eta_{\text {mec }}=\frac{\left(1-\sqrt{\frac{\mu(1+\mu) I}{2}}\right)}{1+\frac{L(1-\mu)(\sqrt[3]{I}+1)^{3}}{\left(1-\sqrt{\frac{2 I \mu}{\mu+1}}\right)}}
$$

Similarly, it's easily seen that there is only one extrema point, with physical meaning, for the efficiency, and it is given by:

$$
x_{m e}=\frac{I \mu+\sqrt{I L \mu(1-\mu)(\sqrt[3]{I}+1)^{3}\left(L(\sqrt[3]{I}+1)^{3}(1-\mu)+1-I \mu\right)}}{I\left(L(\sqrt[3]{I}+1)^{3}(1-\mu)+1\right)}
$$

$$
y_{m e}=z_{m e}=\frac{1}{\sqrt[3]{I}+1}
$$

To see, as above, that the efficiency reaches a maximum, becomes too cumbersome a task if the solution of systems of equations are undertaken. Therefore, an alternative way is presented in that follows, to obtain equation (3.8). And when the efficiency reaches its maximum $\left(x_{m e}, y_{m e}, z_{m e}\right)$ is given by the equations $(3.8,3.9)$.

Indeed, clearly the values of $y_{m e}, z_{m e}$ given by the equation (3.9) fulfill the following two extreme conditions:

$$
\frac{\partial \eta}{\partial y}=0 ; \frac{\partial \eta}{\partial z}=0
$$

Furthermore, as it was seen above, the optimal time ratio and the allocation ratio are the same for both maximum power and ecological function (equations $(3.5,3.6))$. Therefore,

$$
y_{m p}=y_{m e c}=y_{m e}=z_{m p}=z_{m e c}=z_{m e}=\frac{1}{\sqrt[3]{I}+1}
$$

Thus, this values could be included in the equations of power and heat transfer (equations $(2.9,2.11)$ ) and proceed to optimizes the efficiency (equation (2.11) by the following criterion valid when there is only one parameter([10]):

Criterion (Maximum efficiency) Let $\eta=\frac{p}{q_{H}} \quad$ Suppose $\left.\frac{\partial^{2} p}{\partial x^{2}}\right|_{x}=\left.\frac{\partial^{2} q_{H}}{\partial x^{2}}\right|_{x}$, for some $x$. Then the maximum 
efficiency $\eta_{\max }$ is given by

$$
\eta_{\max }=\frac{\left.\frac{\partial p}{\partial x}\right|_{x_{m e}}}{\left.\frac{\partial q_{H}}{\partial x}\right|_{x_{m e}}}
$$

where $x_{m e}$ is the point in which $\eta$ achieves a maximum value.

Then, by the equations (2.9) and (2.10) we obtain the relationships of $p$ and $q_{H}$ with respect to $x$.

$$
\begin{gathered}
p=\frac{(1-I x)\left(1-\frac{\mu}{x}\right)}{(\sqrt[3]{I}+1)^{3}} \\
q_{H}=\frac{\left(1-\frac{\mu}{x}\right)}{(\sqrt[3]{I}+1)^{3}}+L(1-\mu)
\end{gathered}
$$

The conditions of the criterion are clearly satisfied. Indeed,

$$
\frac{\partial^{2} p}{\partial x^{2}}=\frac{\partial^{2} q_{H}}{\partial x^{2}}=-\frac{2 \mu}{x^{3}(\sqrt[3]{I}+1)^{3}}<0
$$

since $x>0$.Therefore (equation(3)),

$$
\eta_{\max }=1-\frac{x_{m e}^{2} I}{\mu}
$$

where $x_{m e}$ must, by the second law, satisfies the inequality ([10]):

$$
\frac{\mu}{I} \leq x_{m e} \leq \sqrt{\frac{\mu}{I}}
$$

if we apply the preceding statement and the equation (3.11), the following inequality is obtained

$$
\eta_{m p}=1-\sqrt{I \mu} \leq \eta_{\max } \leq 1-I \mu=\eta_{C I}
$$

where $\eta_{m p}=1-\sqrt{I \mu}$ and $\eta_{C I}=1-I \mu$ corresponding to (Curzon-Ahlborn)-like and Carnot-like efficiencies; which includes the internal irreversibilities in the $I$ factor.

Nevertheless, we can calculate easily $x_{m e}$ from the following cubic equation:

$$
1-\frac{x_{m e}^{2} I_{S}}{\mu}=\frac{\left.p\right|_{x_{m e}}}{\left.q_{H}\right|_{x_{m e}}}=\frac{\left(1-x_{m e} I\right)\left(1-\frac{\mu}{x_{m e}}\right)}{\left(1-\frac{\mu}{x_{m e}}\right)+L(1-\mu)(\sqrt[3]{I}+1)^{3}}
$$

In solving this equation and taking into account the inequality (3.12), we obtain the equation (3.8).

Finally, the maximum efficiency $\eta_{\max }$ is given by (equation (3.11)):

$$
\eta_{\max }=1-\left(\frac{\left.\sqrt{I \mu}+\sqrt{L(1-\mu)(1+\sqrt[3]{I})^{3}\left(L(1-\mu)(1+\sqrt[3]{I})^{3}+1-I \mu\right.}\right)}{1+L(1-\mu)(\sqrt[3]{I}+1)^{3}}\right)^{2}
$$

The behavior of the efficiencies $\eta_{m p}, \eta_{\text {mec }}$ and $\eta_{\max }$ is shown in the Fig. 2.

In general it has been supposed that $I \geq 1$; but sometimes can be considered that $I=1$. In this case the internal irreversibilities can be physically interpreted as part of the engine's heat leak that brings us to the engine modeled in [2] and [13]. So, substitution of $I=1$ into equations (3.1, 3.5, 3.8) and (3.9) gives:

$$
\begin{aligned}
& x_{m p}=\sqrt{\mu} ; x_{m e c}=\sqrt{\frac{\mu(1+\mu)}{2}} ; \\
& x_{m e}=\frac{\mu+\sqrt{8 L \mu(1-\mu)(8 L(1-\mu)+1-\mu)}}{8 L(1-\mu)+1} \\
& y_{m p}=y_{m e c}=y_{m e}=z_{m p}=z_{m e c}=z_{m e}=\frac{1}{2}
\end{aligned}
$$

The equations:

$$
x_{m p}=\sqrt{\mu} \text { and } z_{m p}=\frac{1}{2}
$$

are the same as the presented in [13] and

$$
x_{m p}=\sqrt{\mu} \text { and } y_{m p}=\frac{1}{2}
$$

corresponding to the CA-engine. Further, the following results are obtained (see equations $(3.3,3.7,3.14)$ ):

$$
\begin{gathered}
\eta_{m p}=\frac{1-\sqrt{\mu}}{1+\frac{8 L(1-\mu)}{1-\sqrt{\mu}}} \\
\eta_{\text {mec }}=\frac{1-\sqrt{\frac{\mu(1+\mu)}{2}}}{1+\frac{8 L(1-\mu)}{\left(1-\sqrt{\frac{2 \mu}{\mu+1}}\right)}}
\end{gathered}
$$

$\eta_{\max }=1-\left(\frac{\sqrt{\mu}+\sqrt{8 L(1-\mu)(8 L(1-\mu)+1-\mu)}}{1+8 L(1-\mu)}\right)^{2}$ 


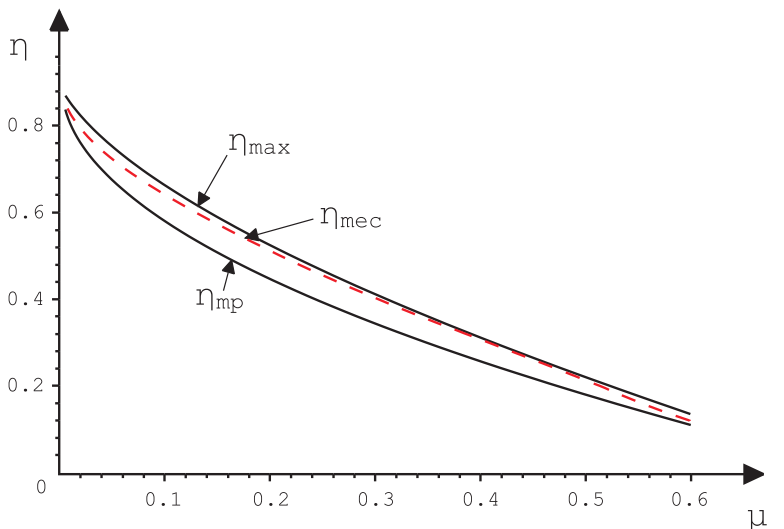

FIG. 2: Graphics of the efficiencies $\eta_{\text {mp }}, \eta_{\text {mec }}$ and $\eta_{\text {max }}$ versus $\mu$ when $I=1.235$ and $L=0.01$.

some of these results have been reported in the literature [6].

On the other hand, the optimization performed in this work gives results that could be applied to the design of power plants. For instance, in the third section it is found that for

$y_{m p}=y_{m \sigma}=y_{m e c}=y_{m e}=z_{m p}=z_{m \sigma}=z_{m e c}=z_{m e}=\frac{1}{\sqrt[3]{I}+1}$

the engine operates at maximum power, efficiency and ecological function and entropy generation local minimum. Therefore, the time rate in the isothermal processes satisfies:

$$
\frac{t_{L}}{t_{H}}=\sqrt[3]{I} \geq 1
$$

This result generalizes to one presented in [2] and it is different to one found in [18]. And when $I=1$,

$$
t_{L}=t_{H}
$$

Similarly, it follows from the equation (3.15) that when the engine operates at maximum power, efficiency and ecological function, the relation for the heat transfer areas for the cold side to the hot side, is:

$$
\frac{A_{L}}{A_{H}}=\sqrt[3]{I} \geq 1 ; \frac{\beta}{\alpha}=\frac{U A_{L}}{U A_{H}}=\sqrt[3]{I}
$$

This result shows that the size of the heat exchanger in the cold side must be larger than the size of heat exchanger in the hot side. Thus, in accordance with the definitions adopted for the thermal conductance, if $I>1$ the one for the cold side results greater than the hot side. Furthermore, if $I=1$

$$
A_{L}=A_{H}
$$

which implies that the allocation of the heat exchangers is balanced ([13]) .

By the equations $(3.16,3.17)$ we have:

$$
\frac{t_{L}}{t_{H}}=\frac{A_{L}}{A_{H}}
$$

which is satisfied when the heat engine operates to maximum power, ecological function and efficiency, and minimum entropy generation. In [17] the above relationship was obtained, by a double optimization of power and efficiency. For $I>1$, the irreversibility produces an inverse relationship between the total area and the total contact time; that is, a less time is needed to transfer the heat that the engine processes. This is due to the fact that less heat goes through the engine. Part of the heat is lost because of internal irreversibility. For $I=1$, the relationship between area and contact time is inversely proportional; that is, if the area is augmented the time is reduced. This result does not depend explicitly of $I$ and differs of one presented in [4] and [2].

\section{A COST AND EFFECTIVENESS OPTIMIZATION.}

A more detailed model would involve acknowledgement that the cost of providing the same heat transfer capability differs between the cold and hot sides. Let this represented as having a cost per unit heat transfer to be $c_{L}$ on the cold side but $c_{H}$ on the hot side ([14]). Then

$$
c_{H} \alpha+c_{L} \beta=C
$$

where $C$ is fixed total cost. Thus we have that the third characteristic parameter $z$ changes to:

$$
z^{*}=\frac{c_{H} \alpha}{C} ; 1-z^{*}=\frac{c_{H} \beta}{C}
$$

In including the optimal values $x_{m p}, y_{m p}$ (equations $(3.1,3.2)$ ) in the equation (2) the dimensionless power $p^{*}=\frac{W}{C^{*} T_{H} t_{H}}$ is given by:

$$
p^{*}=\frac{(1-\sqrt{I \mu})^{2}}{\frac{1}{z^{*}}+\frac{c I^{\frac{2}{3}}}{1-z^{*}}}
$$

where $c=\frac{c_{L}}{c_{H}}>1$ (equation (3.17)) and $C^{*}=\frac{C}{c_{H}}$. The efficiency is given by:

$$
\eta^{*}=\frac{(1-\sqrt{I \mu})^{2}}{1-\sqrt{I \mu}+L(1-\mu)(\sqrt[3]{I}+1)\left(\frac{1}{z^{*}}+\frac{c I^{\frac{2}{3}}}{\left(1-z^{*}\right)}\right)}
$$

In optimizing the power with respect to $z^{*}$, we have:

$$
z_{m p}^{*}=\frac{1}{1+\sqrt{c} \sqrt[3]{I}}
$$

or equivalently

$$
\frac{\beta}{\alpha}=\frac{\sqrt[3]{I}}{\sqrt{c}}
$$

Of course this reverts to the earlier form (equation (3.17)) if $c=1$. 


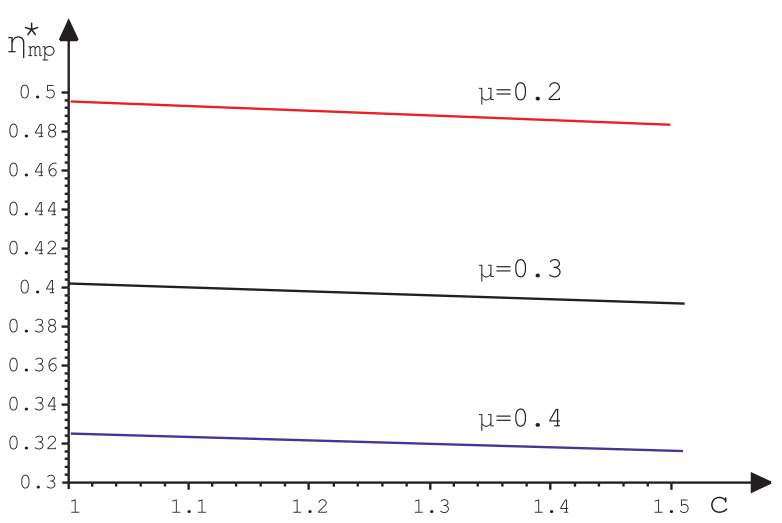

FIG. 3: Graphics of the efficiency $\eta_{m p}^{*}$ versus $c(\mu=0.2,0.3,0.4)$ when $I=1.235$ and with $L=0.01$.

The efficiency that develops maximum power is:

$$
\eta_{m p}^{*}=\frac{1-\sqrt{I \mu}}{1+\frac{L(1-\mu)(1+\sqrt[3]{I})(1+\sqrt[3]{I} \sqrt{c})^{2}}{1-\sqrt{I \mu}}}
$$

The Fig. 3 shows the behavior of $\eta_{m p}^{*}$ versus $c$.

In general, there are two design rules for heat exchange at the two ends of the heat engine ([19]). The first rule is that the thermal conductance is constrained:

$$
\alpha+\beta=\gamma
$$

where $\gamma$ is a constant; which was applied herein for the allocation of the heat exchangers (see equation (2.8) with $\gamma=U A$ ).

The second rule is that the total is constrained by

$$
A=A_{H}+A_{L}
$$

where $A_{H}, A_{L}$ are heat transfer areas on hot and cold side.

To apply the second rule, we may be faced with an existing heat exchange apparatus which is to be redistributed between hot and cold sides to achieve maximum power. Now, the total area $A$ is fixed but when distributed it has different overall heat transfer coefficients and hence different effectiveness on hot and cold sides. Thus,

$$
A=A_{H}+A_{L}=\frac{\alpha}{U_{H}}+\frac{\beta}{U_{L}}
$$

where $U_{H}, U_{L}$ are overall heat transfer coefficients on hot and cold side. In parametrizing again.

$$
z^{* *}=\frac{\alpha}{U_{H} A} ; 1-z^{* *}=\frac{\beta}{U_{L} A}
$$

Again, including the optimal values $x_{m p}, y_{m p}$ (equations (3.1, $3.2)$ ) in the equation (2.5) the dimensionless power $p^{* *}=$ $\frac{W}{A U_{H} t_{H} T_{H}}$ is given by:

$$
p^{* *}=\frac{(1-\sqrt{I \mu})^{2}}{\frac{1}{z}+\frac{I^{\frac{2}{3}}}{(1-z) U}}
$$

where $U=\frac{U_{L}}{U_{H}}$. And the efficiency is now given by:

$$
\eta^{* *}=\frac{(1-\sqrt{I \mu})^{2}}{1-\sqrt{I \mu}+L(1-\mu)(\sqrt[3]{I}+1)\left(\frac{1}{z}+\frac{I^{\frac{2}{3}}}{(1-z) U}\right)}
$$

In optimizing the power with respect to $z^{* *}$, we have:

$$
z_{m p}^{* *}=\frac{\sqrt{U}}{\sqrt[3]{I}+\sqrt{U}}
$$

or equivalently

$$
\frac{\beta}{\alpha}=\sqrt[3]{I} \sqrt{U} ; \quad \frac{A_{L}}{A_{H}}=\frac{\sqrt[3]{I}}{\sqrt{U}}=\sqrt[3]{I} \sqrt{\frac{U_{H}}{U_{L}}}
$$

Then, the optimal distribution of the heat exchangers areas is:

$$
\begin{aligned}
A_{H} & =\frac{A}{1+\sqrt[3]{I} \sqrt{\frac{U_{H}}{U_{L}}}} ; \\
A_{L} & =\frac{A}{1+\frac{1}{\sqrt[3]{I}} \sqrt{\frac{U_{L}}{U_{H}}}}
\end{aligned}
$$

This result has been reported by [20] (when $I=1$ ) using another thermoeconomic criterion. However, it differs when $I \neq 1[21]$.

Then, the efficiency that develops maximum power is:

$$
\eta_{m p}^{* *}=\frac{1-\sqrt{I \mu}}{1+\frac{L(1-\mu)(\sqrt[3]{I}+1)\left(1+\frac{\sqrt[3]{I}}{\sqrt{U}}\right)^{2}}{1-\sqrt{I \mu}}}
$$

The Fig. 4 shows the behavior of $\eta_{m p}^{* *}$ versus $U$.

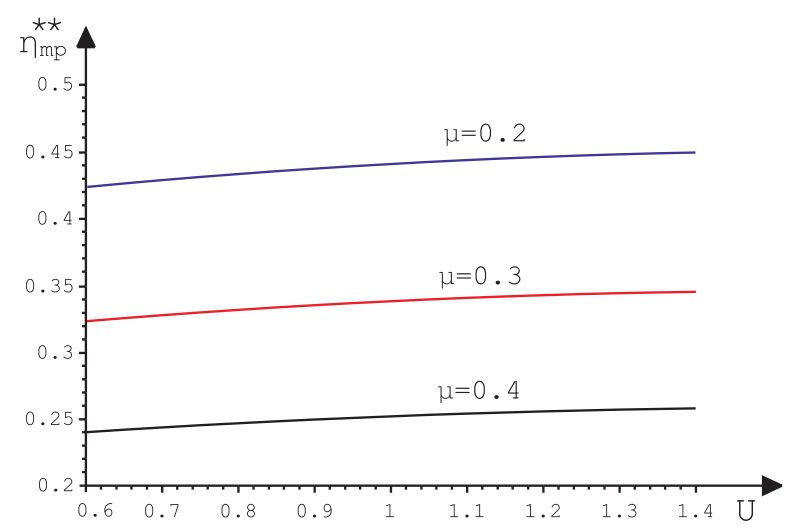

FIG. 4: Graphics of the efficiencies $\eta_{m p}^{* *}$ versus $U(\mu=0.2,0.3,0.4)$ when $I=1.235$ and with $L=0.01$.

\section{CONCLUSIONS}

In the above section, the optimization has been carried for one objective function, that is, the power developed, respect to 
two additional parameters. These parameters could be treated as economic ones. Moreover, the obtained values, also optimizes the other considered objective functions. Such as, entropy generation, ecological function and efficiency. This is due to the fact that the analyzed Carnot engine satisfy the following partial criterion:

Criterion If $f_{i}=f_{i}\left(x, z, z^{*}, z^{* *} \ldots\right)$ represents one of the four objective functions, that is, power, efficiency, ecological function or entropy generation; with $x$ as the internal temperature ratio; $z, z^{*}, z^{* *} \ldots$ are the characteristiceconomic parameters of Carnot cycles belonging to the class of Carnot irreversible cycles analyzed. Moreover, if $z_{m j}, z_{m j}^{*}, z_{m j}^{* *} \ldots$; are the optimum values for functions $f_{j}$ for some $j$, then $z_{m j}, z_{m j}^{*}, z_{m j}^{* *} \ldots$ are also the optimum values for the functions $f_{i}$, for $i \neq j$.

It is a fact that it suffices to develop the optimization for a couple of objective functions, say the power and the efficiency to obtain the parameters that optimizes the remaining objective functions.

For instance, the power and the efficiency satisfy the following functional relationship (equation (2.11)):

$$
\eta(x, \phi)=\frac{p(x, \phi)}{A+g(x) p(x, \phi)}
$$

with $A=L(1-\mu) \neq 0$ (i.e. there is leak heat) and $g(x)=\frac{1}{1-I x}$. Let $\phi=z, z^{*}, z^{* *} \ldots$ Then,

$$
\frac{\partial \eta(x, \phi)}{\partial \phi}=\frac{A \frac{\partial p(x, \phi)}{\partial \phi}}{(A+g(x) p(x, \phi))^{2}}
$$

Therefore,

$$
\left.\frac{\partial \eta}{\partial \phi}\right|_{\phi_{m p}=\phi_{m e}}=\left.0 \Longleftrightarrow \frac{\partial p}{\partial \phi}\right|_{\phi_{m p}=\phi_{m e}}=0
$$

This implies that their roots are the same (necessary condition). It is easily seen that for $\phi_{m p}=\phi_{m e}$, the power and the efficiency reach a maximum (sufficiency condition) since

$$
\left.\frac{\partial^{2} \eta}{\partial \phi^{2}}\right|_{\phi_{m p}=\phi_{m e}}=\frac{\left.A \frac{\partial^{2} p}{\partial \phi^{2}}\right|_{\phi_{m p}=\phi_{m e}}}{(A+g p)^{2}}
$$

A remarkable conclusion of this work is that it is sufficient to find the extreme of some of the functions $f_{i}$, say the power so that

$$
\frac{\partial p}{\partial x}=0 ; \frac{\partial p}{\partial \phi}=0
$$

where $\phi=z, z^{*}, z^{* *} \ldots$ and then substitute in the appropriate functional relationship (for the efficiency is equation(5)) the values of $\phi_{m p}=\phi_{m e}$. The obtained $f_{i}=f_{i}(x)(\eta=\eta(x))$ are then optimized respect to the $x$ parameter only. It is found that the result optimizes the other objective functions.

In other words, for the class of irreversible Carnot engines considered in this work, the $x$ parameter could be considered as the fundamental characteristic parameter of the engine. This is the only parameter that changes its optimal value according to the engine operation conditions. The remaining parameters maintain their optimal value independently of the operation condition of the engine.

Finally, the above mentioned criteria could be applied and extended to other models of irreversible engines [6]. Further work is underway.

\section{Acknowledgement}

This work was supported by the Program for the Professional Development in Automation, through the grant from the Universidad Autónoma Metropolitana and Parker Haniffin - México.
[1] F. L. Curzon and B. Ahlborn, Am. J. Phys. 43, 22 (1975).

[2] K. H. Hoffman, J. M. Burzler, and S. Shuberth, J. Non-Equilib. Thermodyn. 22 (4), 311 (1997).

[3] J. M. Gordon, J. Appl. Phys. 69, 1 (1991).

[4] J. Chen, J. Phys. D: Appl. Phys. 27, 1144 (1994).

[5] Z. Yan and L. Chen, J. Phys. A: Math. Gen. 28, 6167 (1995).

[6] A. Durmayaz, O. S. Sogut, B. Sahin, and H. Yavuz, Progr. Energ. and Combus. Sci. 30, 175 (2004).

[7] L. A. Arias-Hernández, G. Ares de Parga, and F. AnguloBrown, Open Sys. \& Information Dyn. 10, 351 (2003).

[8] A. Calvo, A. Medina, J. M. Roco, J. A. White, and S. Velasco, Phys. Rev. E 63, (4) 0371021 (2001).

[9] Lingen Chen, Xiaoqin Zhi, Fengrui Sun, Chih Wu, A. Open Sys. \& Information Dyn. 10, 351 (2004).

[10] G. Aragón-González, A. Canales-Palma, A. León-Galicia, and M. Musharrafie-Martínez, J. Phys. D: Appl. Phys. 36, 280 (2003).

[11] M. H. Rubin, Phys. Rev. A 15, 2094 (1979).
[12] F. Angulo-Brown. J. Appl. Phys. 69, (11) 7465 (1991).

[13] A. Bejan . Entropy Generation Minimization. CRC Press, Boca Raton, FL (1996).

[14] J. D. Lewins, Int. J. Mech. Eng. Edu. 28 (1), 41 (2000).

[15] P. Y. Papalambros and D. J. Wilde . Principles of optimal design. Cambridge: University Press, p. 142 (2000).

[16] L. Chen, J. Zhou, F. Sun, and C. Wu, A. Appl. Energ. 77, 327 (2004).

[17] G. Aragón-González, A. Canales-Palma, A. León-Galicia, and J. R. Morales-Gómez, Rev. Mex. Fis. 52 (4), 309 (2006).

[18] B. Andresen, P. Salomon, and R. S. Berry, J. Chem. Phys. 66 (4), 1571 (1977)

[19] M. M. Salah EL-Din, Energ. Convers. Manage. 42, 189 (2001).

[20] B. Sahin and A. Kodal, Energ. Convers. Manage. 42, 1085 (2001).

[21] B. Sahin and A. Kodal, Int. J. Ther. Sci. 42, 777 (2003). 